\title{
CESÀRO SUMMABILITY OF THE CONJUGATE SERIES AND THE DOUBLE HILBERT TRANSFORM
}

\author{
JOHN O. BASINGER ${ }^{1}$
}

ABSTRACT. If $f(x, y)$, a $2 \pi$ periodic function in each variable, has a modulus of continuity $w_{f}(\delta)=o(1 / \log (1 / \delta))$ then

$$
\begin{aligned}
& \tilde{\sigma}_{n}(x, y, f) \\
& -\int_{1 / n}^{\pi} \int_{1 / n}^{\pi} \frac{[f(x+u, y+v)-f(x-u, y+v)-f(x+u, y-v)+f(x-u, y-v)]}{4 \tan (u / 2) \tan (v / 2)} d u d v \\
& \rightarrow 0 \text { uniformly in }(x, y)
\end{aligned}
$$

where $\tilde{\sigma}_{n}(x, y, f)$ is the first arithmetic mean of the conjugate series. This theorem is best possible in that $o(1 / \log (1 / \delta))$ cannot be replaced by $O(1 / \log (1 / \delta))$.

Given a $2 \pi$ periodic function $f(x, y)$ we shall define $\tilde{f}(x, y)$, the conjugate of $f(x, y)$ with respect to the double Hilbert transform, to be

$\lim _{\varepsilon, \eta \rightarrow 0}$

$$
\frac{1}{\pi^{2}} \int_{e}^{\pi} \int_{\eta}^{\pi} \frac{[f(x+u, y+v)-f(x-u, y+v)-f(x+u, y-v)+f(x-u, y-v)]}{4 \tan (u / 2) \tan (v / 2)} d u d v .
$$

In [3, p. 170] K. Sokół-Sokołowski proved that if $f(x, y)$ is $2 \pi$ periodic in each variable and belongs to the class $L^{p}, p>1$, then $\tilde{f}(x, y)$ exists almost everywhere.

In this paper we shall show that if $f(x, y)$ is sufficiently continuous then $\tilde{\sigma}_{n}(x, y, f)$

$-\int_{1 / n}^{\pi} \int_{1 / n}^{\pi} \frac{[f(x+u, y+v)-f(x-u, y+v)-f(x+u, y-v)+f(x-u, y-v)]}{4 \tan (u / 2) \tan (v / 2)} d u d v$ $\rightarrow 0$ uniformly in $(x, y)$,

where $\tilde{\sigma}_{n}(x, y, f)$ is the first arithmetic mean of the conjugate series.

Before we proceed we shall need the following definitions and inequalities. Define

$\tilde{f}_{1 / n}(x, y)$

$=\frac{1}{\pi^{2}} \int_{1 / n}^{\pi} \int_{1 / n}^{\pi} \frac{[f(x+u, y+v)-f(x-u, y+v)-f(x+u, y-v)+f(x-u, y-v)]}{4 \tan (u / 2) \tan (v / 2)} d u d v$

and

Received by the editors August 29, 1974.

AMS (MOS) subject classifications (1970). Primary 42 A40.

${ }^{1}$ This is the second part of my dissertation done under the direction of Victor $L$. Shapiro.

(1) American Mathematical Society 1976 


$$
\begin{gathered}
\tilde{\sigma}_{n}(x, y, f)=\frac{1}{\pi^{2}} \int_{-\pi}^{\pi} \int_{-\pi}^{\pi} f(x+u, y+v) \tilde{\sigma}_{n}(u) \tilde{\sigma}_{n}(v) d u d v \\
=\frac{1}{\pi^{2}} \int_{0}^{\pi} \int_{0}^{\pi}[f(x+u, y+v)-f(x-u, y+v) \\
-f(x+u, y-v)+f(x-u, y-v)] \tilde{\sigma}_{n}(u) \tilde{\sigma}_{n}(v) d u d v
\end{gathered}
$$

where

$$
\begin{aligned}
\tilde{\sigma}_{n}(u) & =\sum_{k=1}^{n}(\sin (k u))(n+1-k) /(n+1) \\
& =\frac{1}{2} \tan (u / 2)-\sin (n+1) u /(n+1)(2 \sin (u / 2))^{2} \\
& =\frac{1}{2} \tan (u / 2)-H_{n}(u)
\end{aligned}
$$

is the first arithmetic means of the conjugate Dirichlet kernel. It is well known that

$$
\begin{gathered}
\tilde{\sigma}_{n}(x) \geqslant 0, \quad 0 \leqslant x \leqslant \pi, \\
\left|\tilde{\sigma}_{n}(x)\right| \leqslant n / 2, \quad\left|\tilde{\sigma}_{n}(x)\right| \leqslant A / x, \quad\left|H_{n}(x)\right| \leqslant A /(n+1) x^{2} .
\end{gathered}
$$

THEOREM 1. Let $f(x, y)$ be a continuous $2 \pi$ periodic function with modulus of continuity $w_{f}(\delta)=o(1 / \log (1 / \delta))$. Then

$$
\lim _{n \rightarrow \infty} \tilde{\sigma}_{n}(a, b, f)-\tilde{f}_{1 / n}(a, b)=0 \text { uniformly in }(a, b) \text {. }
$$

Without loss of generality let $a=0$ and $b=0$. By definition,

$$
\tilde{f}_{1 / n}(0,0)=\frac{1}{\pi^{2}} \int_{1 / n}^{\pi} \int_{1 / n}^{\pi} \frac{[f(x, y)-f(-x, y)-f(x,-y)+f(-x,-y)]}{4 \tan (x / 2) \tan (y / 2)} d x d y
$$

and

$$
\begin{array}{r}
\tilde{\sigma}_{n}(0,0, f)=\frac{1}{\pi^{2}} \int_{0}^{\pi} \int_{0}^{\pi}[f(x, y)-f(-x, y)-f(x,-y)+f(-x,-y)] \\
\cdot \tilde{\sigma}_{n}(x) \tilde{\sigma}_{n}(y) d x d y .
\end{array}
$$

Let

$$
g(x, y)=f(x, y)-f(-x, y)-f(x,-y)+f(-x,-y)
$$

and observe that $g(x, y)$ has a modulus of continuity $w_{g}(\delta) \leqslant 4 w_{f}(\delta)=$ $o(1 / \log (1 / \delta))$. Since $g(x, 0)=0$ for all $x$ and $g(0, y)=0$ for all $y$, we have

$$
|g(x, y)| \leqslant \min (o(1 / \log (1 / x)), o(1 / \log (1 / y))) \text {. }
$$

We can now write

$$
\tilde{\sigma}_{n}(0,0, f)-\tilde{f}_{1 / n}(0,0)=\frac{1}{\pi^{2}} \int_{0}^{\pi} \int_{0}^{\pi} g(x, y) \tilde{\sigma}_{n}(x) \tilde{\sigma}_{n}(y) d x d y
$$

$$
-\frac{1}{\pi^{2}} \int_{1 / n}^{\pi} \int_{1 / n}^{\pi} \frac{g(x, y)}{4 \tan (x / 2) \tan (y / 2)} d x d y
$$

Since 


$$
\begin{aligned}
& \int_{1 / n}^{\pi} \int_{0}^{1 / n}|g(x, t)| \tilde{\sigma}_{n}(x) \tilde{\sigma}_{n}(y) d x d y \\
& \leqslant \int_{1 / n}^{\pi} \int_{0}^{1 / n} o(1 / \log n)(n / 2) \tilde{\sigma}_{n}(y) d x d y \\
& \leqslant o(1 / \log n) \int_{1 / n}^{\pi} \tilde{\sigma}_{n}(y) d y=o(1)
\end{aligned}
$$

and

$$
\begin{aligned}
\int_{0}^{1 / n} \int_{0}^{1 / n}|g(x, y)| \tilde{\sigma}_{n}(x) \tilde{\sigma}_{n}(y) d x d y & \\
& \leqslant o(1 / \log n) \int_{0}^{1 / n} \int_{0}^{1 / n} \frac{n^{2}}{4} d x d y \\
& =o(1 / \log n),
\end{aligned}
$$

we can rewrite (1) as follows:

$$
\begin{aligned}
o(1)+\frac{1}{\pi^{2}} \int_{1 / n}^{\pi} \int_{1 / n}^{\pi} g(x, y)[ & -\left(H_{n}(y) / 2 \tan (x / 2)\right) \\
& \left.-\left(H_{n}(x) / 2 \tan (y / 2)\right)+H_{n}(x) H_{n}(y)\right] d x d y .
\end{aligned}
$$

Choose $0<\alpha<1$ and break the integral in (2) into four parts:

$$
\int_{1 / n^{\alpha}}^{\pi} \int_{1 / n^{\alpha}}^{\pi}+\int_{1 / n^{\alpha}}^{\pi} \int_{1 / n}^{1 / n^{\alpha}}+\int_{1 / n}^{1 / n^{\alpha}} \int_{1 / n^{\alpha}}^{\pi}+\int_{1 / n}^{1 / n^{\alpha}} \int_{1 / n}^{1 / n^{\alpha}} .
$$

The first of these is majorized by

$$
\int_{1 / n^{\alpha}}^{\pi} \int_{1 / n^{a}}^{\pi}|g(x, y)|\left\{\frac{A}{(n+1) y^{2} x}+\frac{A}{(n+1) y x^{2}}+\frac{A}{(n+1)^{2} x^{2} y^{2}}\right\} d x d y .
$$

Setting $G=\max |g(x, y)|$ we can bound (4) with

$$
\begin{aligned}
G A\left[O\left(n^{\alpha} \log n^{\alpha} /(n+1)\right)+O\left(n^{\alpha} \log n^{\alpha} /(n+1)\right)\right. \\
\left.+O\left(n^{2 \alpha} /(n+1)^{2}\right)\right]=o(1) .
\end{aligned}
$$

The second and third regions in (3) are majorized by

$$
\begin{aligned}
\int_{1 / n^{\alpha}}^{\pi} \int_{1 / n}^{1 / n^{\alpha}} w_{g}\left(\frac{1}{n^{\alpha}}\right)\left[\frac{A}{(n+1) y^{2} x}+\frac{A}{(n+1) y x^{2}}\right. & \left.+\frac{A}{(n+1)^{2} x^{2} y^{2}}\right] d x d y \\
= & w_{g}\left(\frac{1}{n^{\alpha}}\right)\left[O\left(n^{\alpha} \log n /(n+1)\right)+O\left(n \log n^{\alpha} /(n+1)\right)\right. \\
& \left.+O\left(n^{\alpha} n /(n+1)^{2}\right)\right] \\
= & o(1) .
\end{aligned}
$$

The fourth region is majorized by 


$$
\begin{aligned}
\int_{1 / n}^{1 / n^{\alpha}} \int_{1 / n}^{1 / n^{\alpha}} w_{g}\left(\frac{1}{n^{\alpha}}\right)\left[\frac{A}{(n+1) y^{2} x}+\frac{A}{(n+1) x y^{2}}\right. & \left.+\frac{A}{(n+1)^{2} x^{2} y^{2}}\right] d x d y \\
= & w_{g}\left(\frac{1}{n^{\alpha}}\right)[O(n \log n /(n+1))+O(n \log n /(n+1)) \\
& \left.+O\left(n^{2} /(n+1)^{2}\right)\right] \\
& o(1) .
\end{aligned}
$$

Therefore

$$
\lim _{n \rightarrow \infty} \tilde{\sigma}_{n}(0,0, f)-\tilde{f}_{1 / n}(0,0)=0 .
$$

In order to show this theorem is best possible, we shall construct a continuous $2 \pi$ periodic function $f$ with modulus of continuity $w_{f}(\delta)=$ $O(1 / \log (1 / \delta))$ for which $\lim _{n \rightarrow \infty} \tilde{\sigma}_{n}(0,0, f)-\tilde{f}_{1 / n}(0,0) \neq 0$.

Let

$$
\begin{gathered}
n_{k}=2^{\left(2^{k}\right)}+1, \\
g_{k}(x)= \begin{cases}1 / \log \left(1 /\left(x-\pi / n_{k}\right)\right), & \pi / n_{k} \leqslant x \leqslant 3 \pi / 2 n_{k} \\
1 / \log \left(1 /\left(2 \pi / n_{k}-x\right)\right), & 3 \pi / 2 n_{k}<x \leqslant 2 \pi / n_{k}, \\
0 & \text { otherwise }\end{cases}
\end{gathered}
$$

and $h(x)=\sum_{k=1}^{\infty} g_{k}(x)$. Since the support of the $g_{k}(x), k=1, \ldots$, are disjoint, the modulus of continuity $w_{h}(\delta)$ of $h(x)$ is equal to $\sup _{k} w_{z_{k}}(\delta)$. But each $g_{k}(x)$ has a modulus of continuity $w_{g_{k}}(\delta)=1 / \log (1 / \delta)$; therefore $w_{h}(\delta)$ $=1 / \log (1 / \delta)$. Next we define

$$
f(x, y)= \begin{cases}h(y) & 0 \leqslant x \leqslant \pi, 0 \leqslant y \leqslant x, \\ h(x), & 0 \leqslant x \leqslant \pi, x<y \leqslant \pi, \\ 0, & -\pi \leqslant x<0, \text { or }-\pi \leqslant y<0, \\ 2 \pi \text { periodic, } & \text { otherwise. }\end{cases}
$$

The function $f(x, y)$ is a continuous $2 \pi$ periodic function with modulus of continuity $w_{f}(\delta)=1 / \log (1 / \delta)$.

Since $f(x, y)$ is 0 for $-\pi \leqslant x<0$ or $-\pi \leqslant y<0$,

$$
\begin{aligned}
\tilde{\sigma}_{n}(0,0, f)-\tilde{f}_{1 / n}(0,0)= & \frac{1}{\pi^{2}} \int_{0}^{\pi} \int_{0}^{\pi} f(x, y) \tilde{\sigma}_{n}(x) \tilde{\sigma}_{n}(y) d x d y \\
& -\frac{1}{\pi^{2}} \int_{1 / n}^{\pi} \int_{1 / n}^{\pi} \frac{f(x, y)}{4 \tan (x / 2) \tan (y / 2)} d y d x .
\end{aligned}
$$

Since $f(x, y) \tilde{\sigma}_{n}(x) \tilde{\sigma}_{n}(y) \geqslant 0$ for $\{(x, y) \mid 0 \leqslant x \leqslant \pi, 0 \leqslant y \leqslant \pi\}$,

$$
\begin{aligned}
\tilde{\sigma}_{n}(0,0, f) & -\tilde{f}_{1 / n}(0,0) \\
& \geqslant \frac{1}{\pi^{2}} \int_{1 / n}^{\pi} \int_{1 / n}^{\pi} f(x, y)\left[\tilde{\sigma}_{n}(x) \tilde{\sigma}_{n}(y)-\frac{1}{4} \cot (x / 2) \cot (y / 2)\right] d y d x .
\end{aligned}
$$


For $\frac{1}{2}<\alpha<1$ we shall break the integration into four regions.

From the proof of Theorem 1 we have that the first of these regions is $O\left(n^{2 \alpha} / n^{2}\right)$. Because $f(x, y)$ is symmetric about the line $y=x$, the integrals of the second and third regions are equal. In the second region

$$
\int_{1 / n^{\alpha}}^{\pi} \int_{1 / n}^{1 / n^{\alpha}} f(x, y)\left[\tilde{\sigma}_{n}(x) \tilde{\sigma}_{n}(y)-\frac{1}{4} \cot (x / 2) \cot (y / 2)\right] d y d x
$$

$$
=\int_{1 / n^{\alpha}}^{\pi} \int_{1 / n}^{1 / n^{\alpha}} h(y)\left[\frac{-H_{n}(x)}{2 \tan (y / 2)}-\frac{H_{n}(y)}{2 \tan (x / 2)}+H_{n}(x) H_{n}(y)\right] d y d x .
$$

Breaking the right-hand side of (5) into three parts we have that the first of these is

$$
\begin{aligned}
&-\int_{1 / n^{\alpha}}^{\pi} \int_{1 / n}^{1 / n^{\alpha}} \frac{h(y) H_{n}(x)}{2 \tan (y / 2)} d y d x \\
& \quad=\int_{1 / n^{\alpha}}^{\pi}-H_{n}(x) d x \int_{1 / n}^{1 / n^{\alpha}} \frac{h(y)}{2 \tan (y / 2)} d y .
\end{aligned}
$$

From its definition $h(0)=0$ and $h(y) \leqslant 1 / \log (1 / y)$. Therefore (6) is majorized by

$$
\begin{aligned}
O\left(n^{\alpha} / n\right) O & \int_{1 / n}^{1 / n^{\alpha}} 1 / y \log (1 / y) d y \\
& =O\left(n^{\alpha} / n\right) O\left(-\log \log n^{\alpha}+\log \log n\right) \\
& =O\left(1 / n^{1-\alpha}\right) .
\end{aligned}
$$

The third part of (5) is majorized by

$$
w_{h}(\pi) \int_{1 / n^{\alpha}}^{\pi} \int_{1 / n}^{1 / n^{\alpha}} \frac{A^{2}}{(n+1)^{2} x^{2} y^{2}} d y d x=O\left(1 / n^{1-\alpha}\right) .
$$

The second part of (5) is

$$
\begin{aligned}
-\int_{1 / n^{\alpha}}^{\pi} \int_{1 / n}^{1 / n^{\alpha}} \frac{h(y) H_{n}(y)}{2 \tan (x / 2)} d y d x & \\
& =-\alpha(O(1)+\log n) \int_{1 / n}^{1 / n^{\alpha}} h(y) H_{n}(y) d y .
\end{aligned}
$$

For $n=n_{k}-1$ where $k>k_{0}(\alpha)$ sufficiently large,

$$
h(y)= \begin{cases}0, & 1 / n \leqslant y \leqslant \pi / n_{k} \\ g_{k}(y), & \pi / n_{k} \leqslant y \leqslant 2 \pi / n_{k} \\ 0, & 2 \pi / n_{k}<y \leqslant 1 / n^{\alpha}\end{cases}
$$

Therefore (7) becomes 


$$
\begin{aligned}
\frac{-\alpha(O(1)+\log n)}{n+1} & \int_{\pi / n_{k}}^{2 \pi / n_{k}} \frac{g_{k}(y) \sin (n+1) y}{(2 \sin (y / 2))^{2}} d y \\
& \geqslant \frac{\alpha(O(1)+\log n)}{n+1} \int_{5 \pi / 4 n_{k}}^{7 \pi / 4 n_{k}} \frac{g_{k}\left(5 \pi / 4 n_{k}\right)(-\sin (5 \pi / 4))}{(2 \sin (y / 2))^{2}} d y \\
& \geqslant \frac{\alpha(O(1)+\log n)(-\sin (5 \pi / 4))}{(n+1) \log \left(4 n_{k} / \pi\right)} \int_{5 \pi / 4 n_{k}}^{7 \pi / 4 n_{k}} \frac{A}{y^{2}} d y \\
& \geqslant c>0 .
\end{aligned}
$$

From symmetry and (8) we have that the 4 th part is

$$
\begin{aligned}
& \int_{\pi / n_{k}}^{2 \pi / n_{k}} \int_{y}^{2 \pi / n_{k}} \\
& \quad+\int_{\pi / n_{k}}^{2 \pi / n_{k}} \int_{2 \pi / n_{k}}^{1 / n^{\alpha}} 2 h(y)\left[\frac{-H_{n}(y)}{2 \tan (x / 2)}-\frac{H_{n}(x)}{2 \tan (y / 2)}+H_{n}(x) H_{n}(y)\right] d x d y .
\end{aligned}
$$

Since $H_{n}(x) \leqslant 0$ for $\pi / n_{k} \leqslant x \leqslant 2 \pi / n_{k}$, both the first integral in (9) and the first term in the second integral are positive. Therefore (9) is greater than

$$
\int_{\pi / n_{k}}^{2 \pi / n_{k}} \int_{2 \pi / n_{k}}^{1 / n^{\alpha}} 2 h(y)\left[\frac{-H_{n}(x)}{2 \tan (y / 2)}+H_{n}(x) H_{n}(y)\right] d x d y
$$

whose modulus is majorized by

$$
O\left(-\log \log \left(2 \pi / n_{k}\right)+\log \log \left(\pi / n_{k}\right)\right)+O(1 / \log n)=o(1) .
$$

Combining the above results gives

$$
\tilde{\sigma}_{n}(0,0, f)-\tilde{f}_{1 / n}(0,0) \geqslant o(1)+c \text { for } n=2^{\left(2^{k}\right)}-1 .
$$

\section{REFERENCES}

1. V. L. Shapiro, Fourier series in several variables, Bull. Amer. Math. Soc. 70(1964), 48-93. MR 28 \#1448.

2. 47 \#2265.

3. K. Sokół-Sokołowski, On trigonometric series conjugate to Fourier series of two variables, Fund. Math. 34(1947), 166-182. MR 9, 89.

4. A. Zygmund, Trigonometric series. Vols. I, II, Cambridge Univ. Press, London and New York, 1968. MR 38 \# 4882.

Department of Mathematics, University of California, Riverside, California 92502 